\title{
Unsuspected iatrogenic occlusion of left main coronary artery
}

\author{
Rajeev Bhardwaj ${ }^{1 *}$, Sanjeev Asotra ${ }^{2}$, Rajeev Marwah ${ }^{2}$ and Ravi Kumar ${ }^{2}$ \\ ${ }^{1}$ Department of Cardiology, MM Institute of Medical Sciences and Research, India \\ ${ }^{2}$ Department of Cardiology, Indira Gandia Medical College, India
}

\begin{abstract}
We present a case, who was being subjected to coronary angiography, as per protocol before aortic valve replacement. Just after left coronary angiography, patient had cardiac arrest. When there was no response to 15 minutes of CPR, check angiography showed left main occlusion at ostium. Patient had successful angioplasty to left main coronary artery
\end{abstract}

\section{Introduction}

Dissection of left main coronary artery (LMCA), is most dreaded complication for any interventional cardiologist. Although rare, with a reported incidence of less than $0.1 \%$ [1]. Its occurrence can have devastating consequences if not promptly treated with immediate revascularization. In its most severe form, it leads to abrupt cessation of blood flow to a large portion of myocardium supplied by left anterior descending (LAD) artery and left circumflex (LCx) artery. This results in pump failure and cardiac arrest. If not treated promptly, it is mostly fatal. Before 1993, when the first successful percutaneous bail-out LMCA stenting was performed, urgent coronary artery bypass surgery $(\mathrm{CABG})$ was the treatment of choice. However, many patients died before even making it to the operation theatre. Hence immediate percutaneous coronary intervention (PCI) seems to be an appropriate and feasible alternative if performed by an experienced interventionalist, as demonstrated by our case.

\section{Case}

48 years male presented to our hospital with history of palpitation for 10 years, and angina class II for last 3 months. His echocardiography showed evidence of biscuspid aortic valve disease with severe aortic regurgitation (AR) with mild aortic stenosis (AS) with normal left ventricular function. His ejection fraction $(\mathrm{EF})$ was $60 \%$. He was advised aortic valve replacement (AVR). As per protocol, surgeons advised coronary angiography, before AVR.

Our resident took the patient for coronary angiography. Left system was found to be normal (Figure 1). Immediately after the left coronary catheter was removed, patient had seizures, hypotension, respiratory arrest followed by cardiac arrest. We started the cardiopulmonary resuscitation (CPR), patient was intubated and temporary pacemaker was inserted. The CPR continued for 15 minutes without any improvement. We then suspected left main dissection. So left Judkin,s guiding catheter was taken for check angiography. We could not hook left main. When we injected contrast in the region of left coronary sinus. We found a pouch like projection in the aorta, around the LMCA origin, but LMCA was not seen (Figure 2). We tried to manipulate floppy wire into LMCA, but it was entering the pouch. After around five

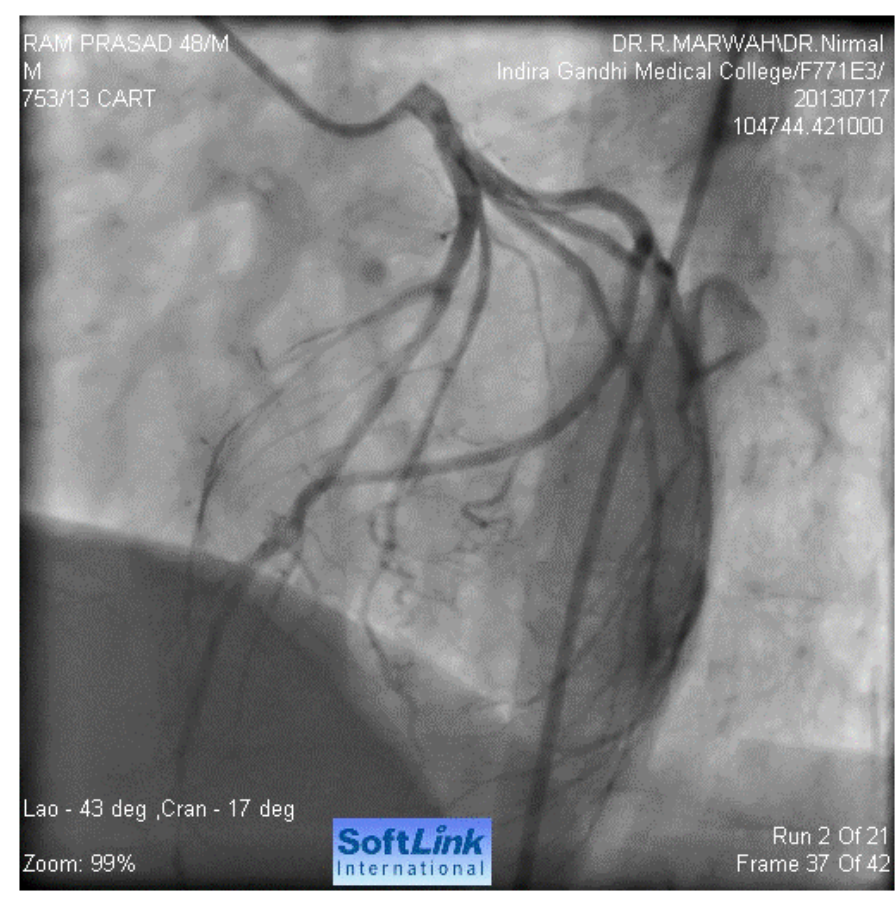

Figure 1. Normal left coronary artery

minutes of effort, with CPR still continuing, we could manage the wire through LMCA to LCx. (Figure 3). We dilated the ostium of LMCA with $2 \mathrm{X} 10 \mathrm{~mm}$ balloon, twice (Figure 4). But there was hardly any flow (Figure 5). Then we implanted 4X12 mm bare metal stent (Figure 6). There was good flow (Figure 7). Immediately electric activity was seen

*Correspondence to: Rajeev Bhardwaj, Department of Cardiology, MM Institute of Medical Sciences and Research, US Club Shimla, 17100, India, E-mail: rajeevbhardwaj_dr@yahoo.com

Keywords: left main coronary artery, dissection, angioplasty

Received: April 28, 2021; Accepted: May 10, 2021; Published: May 21, 2021 


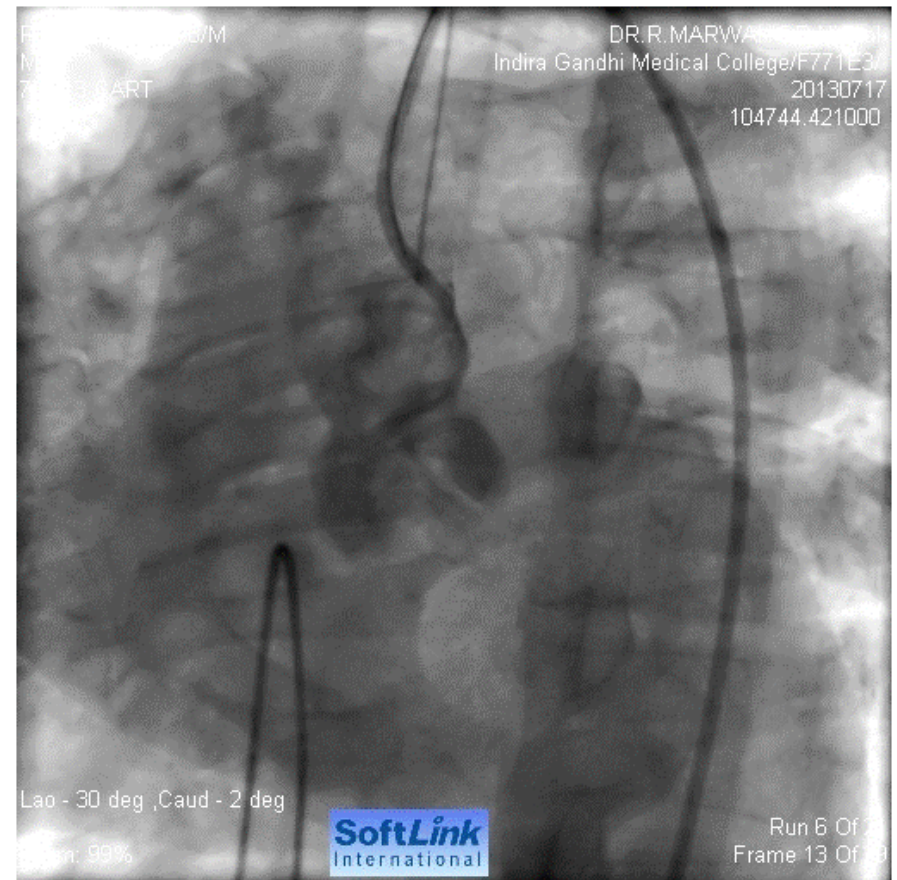

Figure 2. Injection in ascending shows pouch like projection in the region of LMCA LMCA not seen

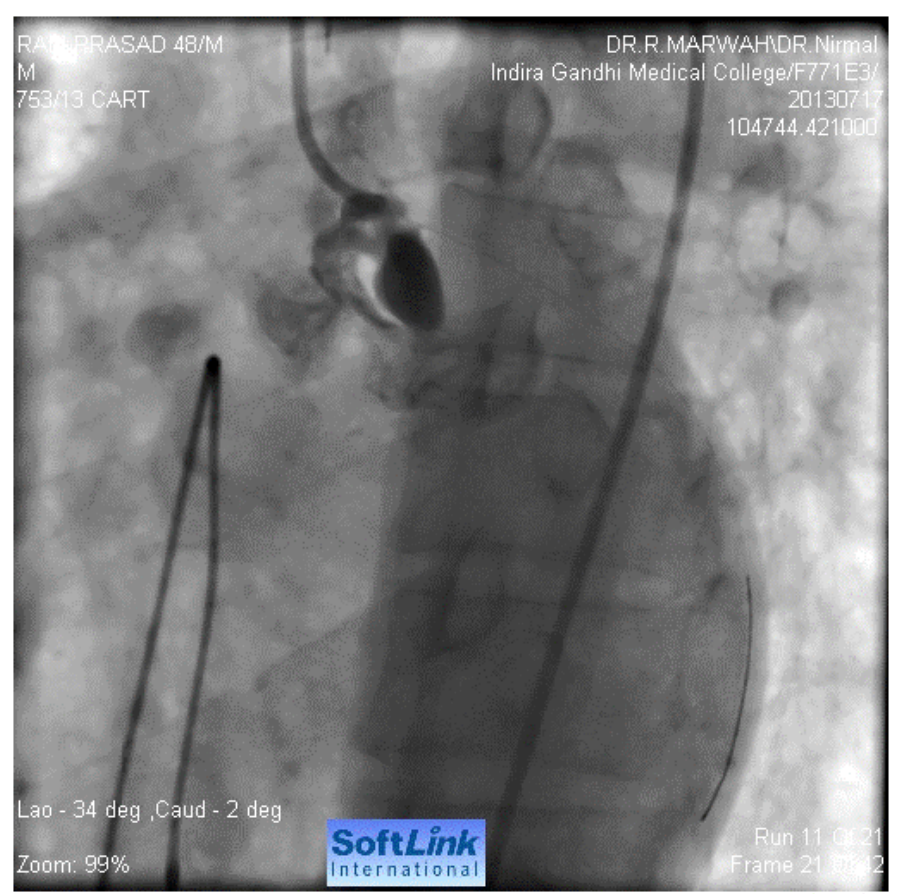

Figure 3. Guide wire across LMCA into LCx

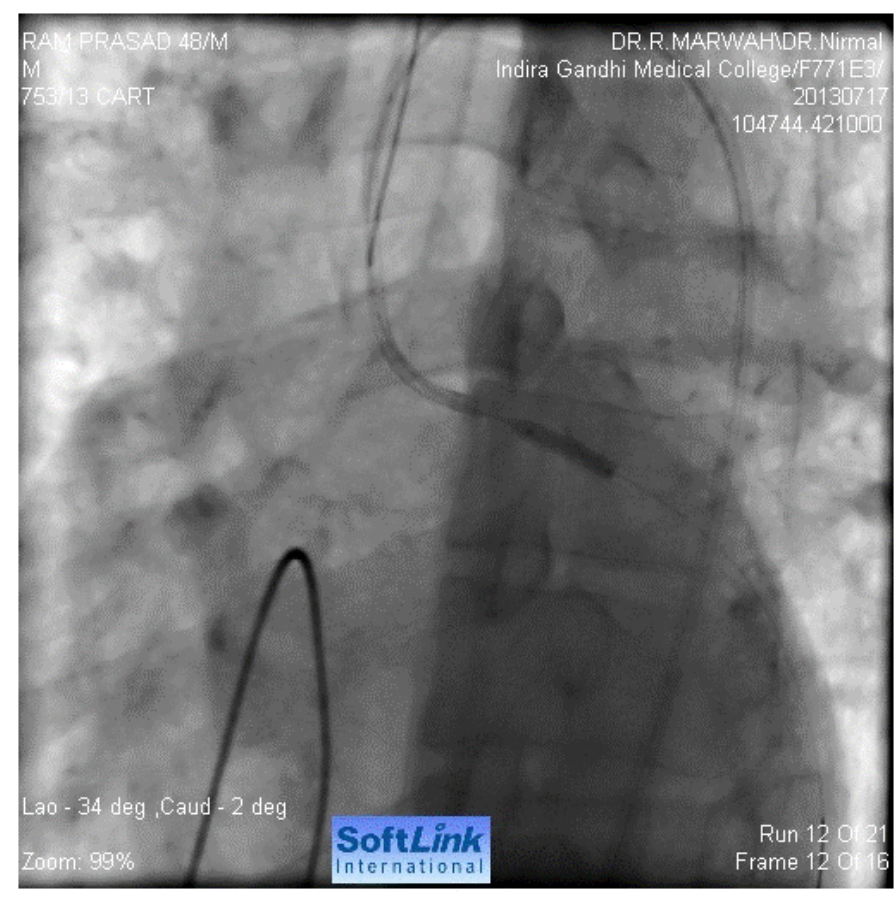

Figure 4. Balloon dilatation in left main ostium

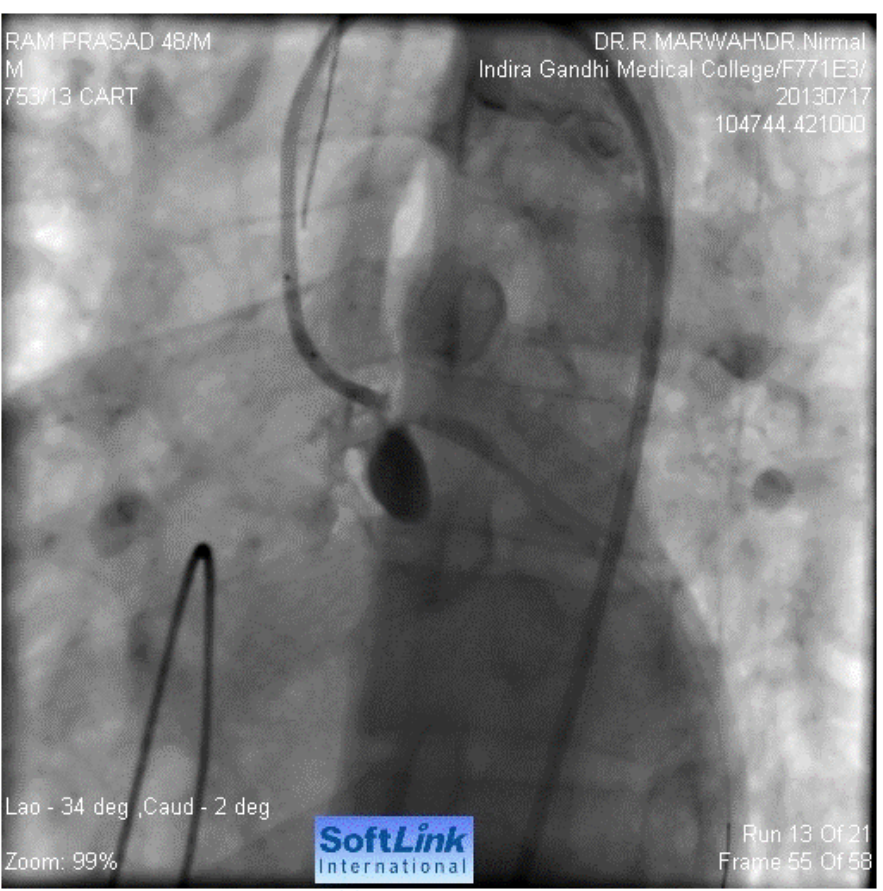

Figure 5. Hardly any flow after balloon dilatation 


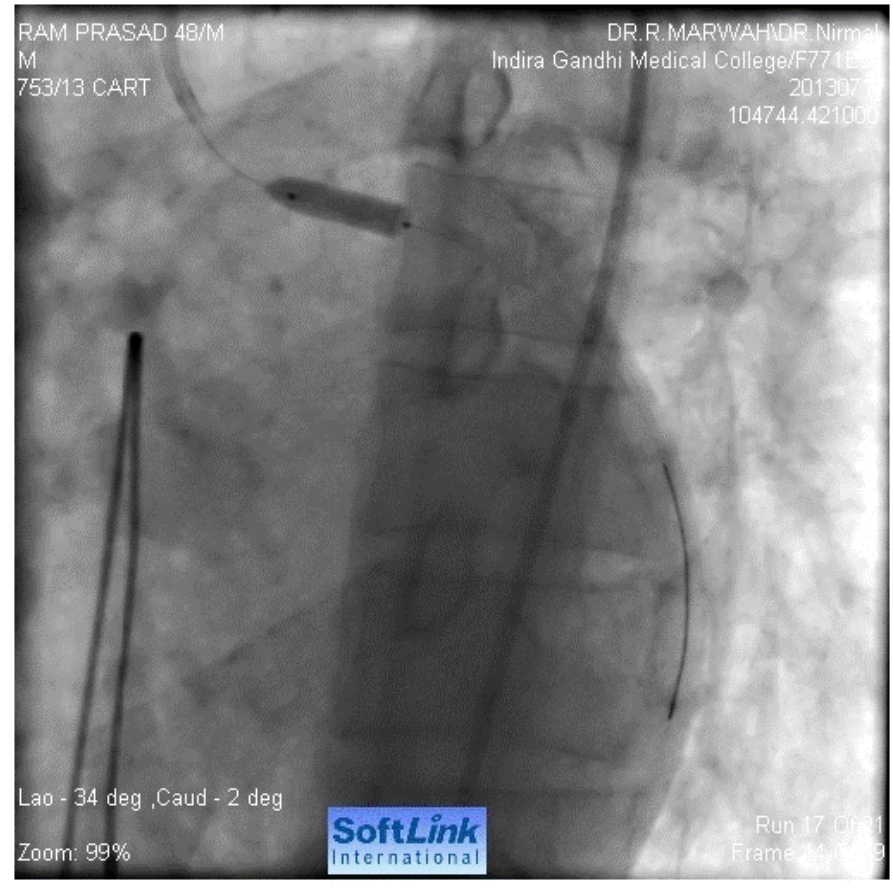

Figure 6. Stent implantation

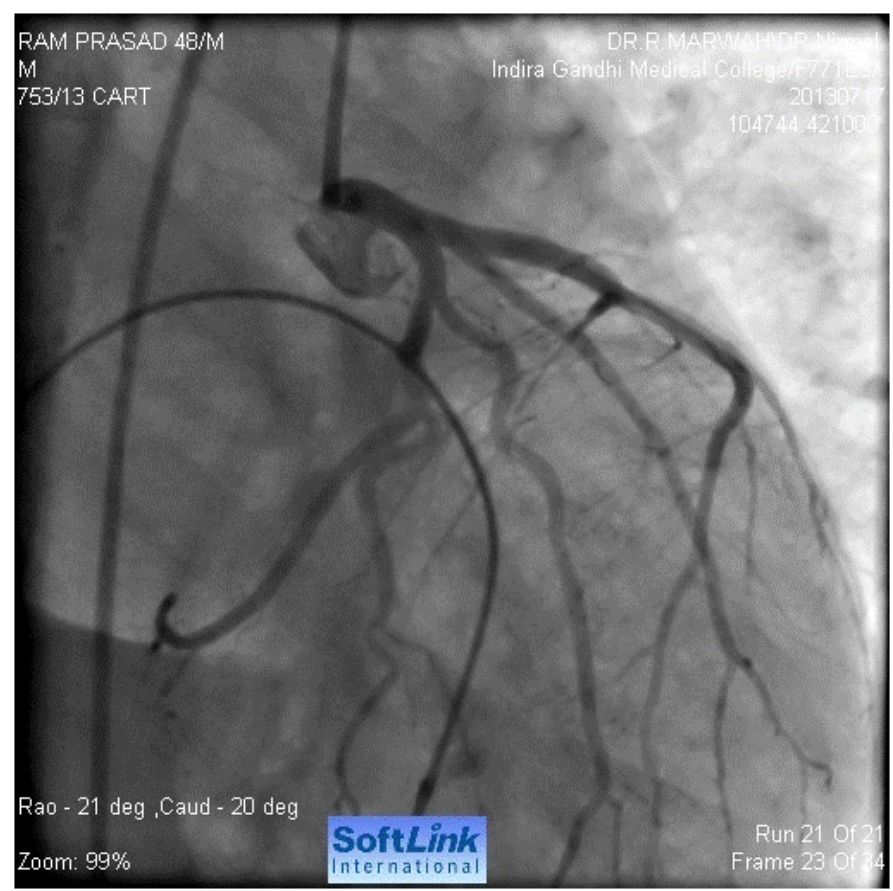

Figure 7. Final result, shows normal flow

on monitor. BP started coming up and was 130/80 after 2 minutes. We observed the patient in the Lab for 10-15 minutes and then shifted him to our cardiac care unit (CCU); still on ventilator and unresponsive to commands and painful stimuli. After 2-3 hours, patient showed movement of limbs. After around 4-5 hours, he started fighting with ventilator and was extubated. He was sitting the next day, fully alert. He was discharged on third day. Check angiography was done after one year. The stent was patent. Right coronary artery was also normal. He underwent successful AVR.

\section{Discussion}

Iatrogenic LMCA dissection results from mechanical injury to the arterial wall during catheter manipulation or passage or deployment of an interventional device. Patients with LM stenosis, hypertension, Marfan syndrome, congenitally unicuspid and bicuspid aortic valves, and cystic medial necrosis have been reported to be at higher risk of dissection [2]. Extensive catheter manipulation, catheter type (e.g., Amplatz catheter, small Judkins catheter resulting in deep LMCA intubation), stiffer and less manageable guide wires (e.g., pressure wire), unusual LMCA anatomy or location, operator experience, and presence of LMCA atherosclerosis have all been associated with an increased risk of dissection $[3,4]$.

In our case, the first operators reported some difficulty and catheter manipulation during LMCA cannulation. They initially suspected contrast reaction and seriousness of the problem was not realized. However, they immediately intubated the patient and CPR was started, inserted temporary pacemaker which prevented brain damage, as LMCA could only be stented more than half an hour after the cardiac arrest.

In a large study of 38 patients with iatrogenic LMCA dissection, an inappropriate position of the diagnostic catheter was responsible for $58 \%$ of the cases [1].

Aorto-coronary dissection should be suspected in any patient undergoing a percutaneous coronary artery intervention who develops otherwise unexplained hypotension and hemodynamic compromise. Once dissection occurs, the clinical picture differs depending on the remaining antegrade coronary flow and can range from an asymptomatic patient with preserved thrombolysis in myocardial infarction (TIMI) grade 3 flow, to a patient in refractory cardiogenic shock whose LMCA is completely compromised. While iatrogenic aorto-coronary artery dissection is obvious on coronary angiography, it may underestimate the extent of aortic involvement. Physical examination findings such as a murmur of aortic regurgitation and diminished peripheral pulses are less prevalent. Even in cases of initially preserved TIMI-3 flow with stable hemodynamics, deterioration may acutely occur because of compromise to flow from a progressive dissection or a superimposed thrombus formation [5]. Hence, every dissection mandates immediate formulation of a good management plan.

Coronary dissections are classified using the National Heart, Lung, and Blood Institute (NHLBI) diagnostic criteria. The NHLBI has six types of dissections classified based on the extent and location of radiolucent areas within the coronary lumen during contrast injection and persistence of contrast. The simplified classification scheme defines three types of coronary dissections involving the left main artery. Type 1 is a localized dissection without extension into the LAD or LCx. Extension of LMCA dissection into LAD or LCX is defined as type 2 , and if extension involves the aortic root then it is type 3 [1]. While type I dissections were associated with excellent outcomes (no hemodynamic instability nor in-hospital death), type 3 dissections had $100 \%$ in-hospital mortality1.

Importance of prompt and timely recognition of this complication to prevent both retrograde and antegrade extension cannot be overemphasized. Conservative therapy, bailout stent implantation and emergent CABG have all been described extensively in literature as potential strategies for the management of iatrogenic aorto-coronary dissection. Given the unpredictable nature of the dissection and the potential for catastrophic sequelae, conservative therapy is rarely considered as a therapeutic option for aorto-coronary dissection. In the 
setting of aorto-coronary dissection and hemodynamic collapse, IABP alone will not provide hemodynamic stability $[6,7]$ and extracorporeal support is not readily available in all centers. Successful treatments (namely percutaneous or surgical) of any arterial dissection require sealing of entry site to thrombose the false lumen and thus prevent progression of vessel dilatation and/or rupture8. Some operators have suggested that for dissections starting downstream and propagating retrograde, the site of initial coronary dissection should be stented first with retrograde stenting of the aorta if needed. While others have proposed immediate ostial stenting irrespective of whether the dissection starts from the ostium or further downstream in the coronary [8]. We think surgery should only be considered on the occasion when stenting fails to seal the dissection or if the aortic valve becomes involved. Alternatively, in patients with dissection induced aortic regurgitation or hemopericardium, immediate surgical intervention should be sought. Dunning et al [9] have suggested that patients be managed by surgery if the dissection extends beyond $40 \mathrm{~mm}$ from the coronary ostium. However, Carstensen et al8, based on their study, suggest immediate ostial stenting even when significant aortic involvement has occurred, as the ostial stent has high changes of successfully sealing the dissection and it does not compromise the chance of a future surgical success.

Our patient was unique in the sense that, here dissection occurred during diagnostic angiography, in a patient with normal coronaries, was type 3 dissection, it was suspected after around 15 minutes of CPR, there was no visible left main ostium, and patient recovered fully after stent implantation. To the best of our knowledge, this is the first survival of such a case in the literature. Koza et el also described one case of occlusion of LMCA following coronary angiography due to plaque rupture, in a case of LMCA disease and managed it with stenting [9].

Several lessons can be learned from this case. First, it obliges us to remember that coronary angiography remains an invasive investigation with rare but life-threatening complications. Indications for diagnostic angiography should therefore be sound. Second, all catheters must be manipulated cautiously, especially when engaging the LMCA. Injections should only be made when catheters are properly placed and when normal pressures have been identified. Third, in the unlucky situation of an iatrogenic LMCA dissection, prompt diagnosis and therapy must be initiated, and the most experienced colleagues and surgeons should be alerted.

\section{References}

1. Eshtehardi P, Adorjan P, Togni M, Tevaearai H, Vogel R, Seiler C, et al. (2010) Iatrogenic left main coronary artery dissection: incidence, classification, management, and long-term follow-up. Am Heart $J$ 159: 1147-1153. [Crossref]

2. Awadalla H, Sabet S, Sebaie A, Rosales O, Smalling R (2005) Catheter-induced lef main dissection incidence, predisposition and therapeutic strategies experience from two sides of the hemisphere. J Invasive Cardiol 17: 233-236. [Crossref]

3. Kovac JD, Bono DP (1996) Cardiac catheter complications related to left main stem disease. Heart 76: 76-78. [Crossref]

4. Slack JD, Pinkerton CA, VanTassel JW, Orr CM (1986) Left main coronary artery dissection during percutaneous transluminal coronary angioplasty. Cathet Cardiovasc Diagn 12: 255-260. [Crossref]

5. Onsea K, Kayaert P, Desmet W, Dubois CL (2011) Iatrogenic left main coronary artery dissection. Neth Heart J 19: 192-195. [Crossref]

6. Lao EP, Nie SP, Ma CS (2013) Immediate bail-out TAP-stenting for the treatment of iatrogenic aortocoronary dissection involving left main bifurcation. J Geriatr Cardiol 10: 202-204. [Crossref]

7. Carstensen S, Ward MR (2008) Iatrogenic aortocoronary dissection: the case for immediate aortoostial stenting. Heart Lung Circ 17: 325-329. [Crossref]

8. Dunning DW, Kahn JK, Hawkins ET, O'Neill WW (2005) Iatrogenic coronary artery dissections extending into and involving the aortic root. Catheter Cardiovasc Interv 51: 387-393. [Crossref]

9. Koza Y, Tas H, Sarac I (2019) Successful management of Iatrogenic left main coronary artery occlusion during coronary angiography- a case report and brief review. Cardiovasc Revasc Med 20: 432-435. [Crossref]

Copyright: $@ 2021$ Bhardwaj R. This is an open-access article distributed under the terms of the Creative Commons Attribution License, which permits unrestricted use, distribution, and reproduction in any medium, provided the original author and source are credited. 